\title{
The Rac1 Inhibitor NSC23766 Suppresses CREB Signaling by Targeting NMDA Receptor Function
}

\author{
Hailong Hou, ${ }^{1}{ }^{\circledR}$ Andrés E. Chávez, ${ }^{3}$ Chih-Chieh Wang, ${ }^{4}$ Hongtian Yang, ${ }^{1}$ Hua Gu, ${ }^{1}$ Benjamin A. Siddoway, ${ }^{1}$ \\ Benjamin J. Hall, ${ }^{4,5}$ Pablo E. Castillo, ${ }^{3}$ and Houhui Xia ${ }^{1,2}$ \\ ${ }^{1}$ Neuroscience Center and ${ }^{2}$ Department of Cell Biology and Anatomy, LSU Health Science Center, New Orleans, Louisiana 70112, ${ }^{3}$ Department of \\ Neuroscience, Albert Einstein College of Medicine, Bronx, New York 10461, and ${ }^{4}$ Neuroscience Program and ${ }^{5}$ Departments of Cell and Molecular Biology, \\ Tulane University, New Orleans, Louisiana 70118
}

\begin{abstract}
NMDA receptor signaling plays a complex role in CREB activation and CREB-mediated gene transcription, depending on the subcellular location of NMDA receptors, as well as how strongly they are activated. However, it is not known whether Racl, the prototype of Rac GTPase, plays a role in neuronal CREB activation induced by NMDA receptor signaling. Here, we report that NSC23766, a widely used specific Racl inhibitor, inhibits basal CREB phosphorylation at S133 (pCREB) and antagonizes changes in pCREB levels induced by NMDA bath application in rat cortical neurons. Unexpectedly, we found that NSC23766 affects the levels of neuronal pCREB in a Rac1-independent manner. Instead, our results indicate that NSC23766 can directly regulate NMDA receptors as indicated by their strong effects on both exogenous and synaptically evoked NMDA receptor-mediated currents in mouse and rat neurons, respectively. Our findings strongly suggest that Rac1 does not affect pCREB signaling in cortical neurons and reveal that NSC23766 could be a novel NMDA receptor antagonist.
\end{abstract}

Key words: antagonist; CREB; NMDA receptor; NSC23766; Rac1

\section{Introduction}

CREB-mediated gene transcription plays important roles in numerous cellular processes as diverse as neuronal plasticity and cell survival (Lyons and West, 2011). Phosphorylation of CREB at serine 133 (pCREB) causes a conformational change in CREB, which allows its interaction with CREB binding protein (CBP; Deisseroth and Tsien, 2002). This CBP interaction can bring CREB to DNA transcriptional machinery promoting gene transcription and protein synthesis (Lyons and West, 2011). Notably, $\mathrm{Ca}^{2+}$ influx through synaptic NMDARs initiates different kinase pathways including MAPK, which phosphorylate CREB at serine 133, leading to CREB activation and CREB-mediated gene transcription critical for neuronal survival (Hardingham and Bading, 2010). However, in contrast to synaptic NMDAR signaling, extrasynaptic NMDAR signaling shuts off pCREB (Hardingham et al., 2002; Hou et al., 2013; Karpova et al., 2013), presumably via

\footnotetext{
Received April 23, 2014; revised Aug. 21, 2014; accepted Sept. 4, 2014.

Author contributions: H.H., B.J.H., P.E.C., and H.X. designed research; H.H., A.E.C., C.C.W., H.Y., H.G., and B.A.S. performed research; H.H., A.E.C., C.-C.W., B.J.H., and H.X. analyzed data; H.H., A.E.C., B.J.H., P.E.C., and H.X. wrote the paper.

This work was supported by funding from National Science Foundation (NSF; IOS-0824393), National Institutes of Health (NIH; R01NS060879), and LSUHSC Research Enhancement Fund (REF) to H.X.; NIH (MH099378) and NSF Career Award (NS065374) to B.J.H.; and NIH (DA017392, MH081935) to P.E.C., A.E.C. was supported by a National Alliance for Research on Schizophrenia and Depression Young Investigator Grant from the Brain and Behavior Research Foundation.

The authors declare no competing financial interests.

Correspondence should be addressed to Dr. Houhui Xia, Neuroscience Center, LSU Health Science Center, New Orleans, LA 70112. E-mail: hxia@|suhsc.edu.

DOI:10.1523/JNEUROSCI.1659-14.2014

Copyright $\odot 2014$ the authors $\quad 0270-6474 / 14 / 3414006-07 \$ 15.00 / 0$
}

activating phosphatase pathways (Colombo et al., 2003) and/or inhibiting kinase pathways (Sala et al., 2000).

Rac1, a member of the Rho family of GTPases, is ubiquitously expressed in mammals and involved in the regulation of many signaling pathways that regulate synaptic plasticity and memory formation (Kim et al., 2007; Haditsch et al., 2009). CREB signaling has also been implicated in synaptic plasticity and memory (Kandel, 2012). Moreover, Rac1-GEF Tiam1, and thus Rac1, can transduce NMDAR activation to cell development (Tolias et al., 2005; Xiao et al., 2013). These studies are consistent with an idea that Racl could couple NMDAR to CREB activation in neurons. NSC23766, a small chemical compound, targets the domain on Racl critical for guanine nucleotide exchange factor (GEF) interaction to inhibit Racl binding and activation by the Rac-specific GEF Trio or Tiam1 (Gao et al., 2004). Thus, when compared with molecular manipulations of Rac1 proteins, NSC23766 emerges as an important practical tool to determine whether Racl is involved in NMDAR signaling to CREB.

Here, using primary cortical neurons, we found that the Racl inhibitor NSC23766 exerts opposite effects on CREB signaling induced by mild versus strong NMDA stimulation. NSC23766 downregulates low-dose NMDA-induced CREB activation, while CREB signaling is activated when it is applied together with high-dose NMDA, which alone induces pCREB shutoff. Unexpectedly, using molecular manipulation of Rac1, we found that NSC23766 regulates pCREB signaling independent of Rac1 function. Moreover, our results show that NSC23766 can directly inhibit NMDAR-mediated currents. While our work excludes a role of Rac1 in CREB signaling, it reveals that NSC23766 could be a novel NMDAR antagonist, which can convert the pCREB shut- 
off induced by strong activation of NMDARs, relevant to glutamate toxicity as seen in stroke, to positive PCREB, and thus beneficial signaling for neuronal health.

\section{Materials and Methods}

Cell cultures for Western blotting experiments. Primary rat cortical neurons were prepared from mixed male/female E18 Sprague Dawley rat embryos. Cells were plated on poly-L-lysine culture dishes in Neurobasal medium supplemented with 2\% B27 and 1\% GlutaMAX (Siddoway et al., 2013). Unless, otherwise stated, 2 -week-old rat cortical neurons were used in the study.

Chemicals and antibodies. These include the following: NSC23766 and bicuculline (Tocris Bioscience), NMDA and okadaic acid (Sigma), antiCREB pS133 and anti-Rac1 (1:1000; Millipore), anti-CREB (1:500; Santa Cruz Biotechnology), anti-GFP (1:1000; Roche), anti-pERK1/2 and ERK1/2 (1:500; Cell Signaling Technology).

Generation of RNAi vectors, recombinant viruses, and infection. pLL3.7based lentivirus was used to express shRNA. The shRNA target for rat Rac1 was GAGGAAGAGAAAATGCCTG. EGFP-tagged Rac1 WT, Rac1 Q61L, and Rac1 T17N (Addgene) were cloned into pSinRep5(nsP2S) vector while recombinant lentiviruses and Sindbis viruses were prepared as reported previously(Hou et al., 2013). Dissociated cortical cultures 13-16 DIV were infected with recombinant Sindbis viruses encoding various Racl proteins for $24 \mathrm{~h}$ before experiment. Neurons (10-12 DIV) were infected with recombinant lentiviruses for $5 \mathrm{~d}$ to knockdown endogenous Rac1.

NMDAR stimulation in Western blotting studies of CREB/ERk1/2 activation. Exogenous application of NMDA was directly applied to the conditioned medium and its effect was measured at different time points (e.g., 2, 5, 10, 30, and $60 \mathrm{~min}$ ). NSC23766 was added to conditioned medium 30 min before (pretreatment), simultaneously (coapplication), or 2 min after (post treatment) NMDA application and was present for the rest of the experiment.

Hippocampal slice electrophysiology. Acute transverse hippocampal slices (400 $\mu \mathrm{m}$ thick) were prepared from Sprague Dawley rats, P21-P30 as previously described (Chávez et al., 2010). Briefly, the hippocampi were isolated and cut in a solution containing the following (in $\mathrm{mM}$ ): 215 sucrose, $2.5 \mathrm{KCl}, 26 \mathrm{NaHCO}_{3}, 1.6 \mathrm{NaH}_{2} \mathrm{PO}_{4}, 1 \mathrm{CaCl}_{2}, 4 \mathrm{MgCl}_{2}, 4 \mathrm{MgSO}_{4}$, and 20 glucose. Thirty minutes post sectioning, slices were incubated in extracellular ACSF recording solution containing the following (in $\mathrm{mM}$ ): $124 \mathrm{NaCl}, 2.5 \mathrm{KCl}, 26 \mathrm{NaHCO}_{3}, 1 \mathrm{NaH}_{2} \mathrm{PO}_{4}, 2.5 \mathrm{CaCl}_{2}, 1.3 \mathrm{MgSO}_{4}$, and 10 glucose, equilibrated with $95 \% \mathrm{O}_{2}$ and $5 \% \mathrm{CO}_{2}, \mathrm{pH}$ 7.4. Experiments were performed at $28 \pm 1^{\circ} \mathrm{C}$ in a submersion-type recording chamber perfused at $\sim 1-2 \mathrm{ml} / \mathrm{min}$ with ACSF containing $10 \mu \mathrm{M}$ NBQX and 100 $\mu \mathrm{M}$ PTX, to block AMPA/kainate and GABA receptors, respectively. Whole-cell patch-clamp recordings were made from CA1 pyramidal neurons voltage clamped at $+40 \mathrm{mV}$ using patch-type pipette electrodes $(\sim 3-4 \mathrm{M} \Omega$ ) containing the following (in $\mathrm{mm}$ ): $131 \mathrm{Cs}-$ Gluconate, 8 $\mathrm{NaCl}, 1 \mathrm{CaCl}_{2}, 10$ EGTA, 10 glucose, and 10 HEPES; pH 7.4, 285-292 $\mathrm{mmol} / \mathrm{kg}$. NMDAR-mediated EPSCs were evoked using monopolar stimulation with a patch-type pipette filled with ACSF and placed in the stratum radiatum (SR). Extracellular fEPSPs were performed using recording pipettes filled with $1 \mathrm{M} \mathrm{NaCl}$ and stimulating patch-type pipette placed in the SR. NSC23766 was bath applied after establishment of a stable baseline $(\sim 10-15 \mathrm{~min})$, and effects were measured after responses reached a new steady state (typically $>15 \mathrm{~min}$ ). LTP was induced by theta-burst stimulation, which consisted of a series of 10 bursts of five stimuli (100 Hz within the burst, $200 \mathrm{~ms}$ interburst interval) repeated four times ( $5 \mathrm{~s}$ apart), whereas LTD was induced using a LFS protocol (900 pulses, $1 \mathrm{~Hz}$ ). For these experiments, slices were incubated in 100 $\mu \mathrm{M}$ NSC23766 for at least $30 \mathrm{~min}$ before induction protocol. Drugs were obtained from Sigma and Tocris Bioscience. Statistical comparisons were made using paired Student's $t$ test and ANOVA ( $p<0.05$ significance), and using OriginPro 8.6 software. Unless otherwise indicated, all values are provided as mean \pm SEM and illustrated traces are averages of 31-40 responses.

Recording of total NMDAR-mediated currents in mouse cortical cultures. Cortices were dissected from mixed male/female E16 mice harvested from pregnant female mice. Detailed methods and procedures were described previously (Hall et al., 2007; Wang et al., 2011). Briefly, cells were dissociated, triturated, and maintained in low-serum media at $37^{\circ} \mathrm{C}$ under $5 \%$ ambient $\mathrm{CO}_{2}$. Cultures of neurons were plated, grown, and maintained in parallel. At 11 DIV, neurons from each genotype were removed for recording. Cells were constantly perfused at room temperature with ACSF at $1 \mathrm{ml} / \mathrm{min}$ and visualized at $40 \times$ under DIC optics. Visually identified pyramidal neurons were patched in a whole-cell voltage-clamp configuration. NMDAR-mediated currents were evoked using short $(10-75 \mathrm{~ms})$ and localized $(50 \mu \mathrm{m})$ pressure-driven application of NMDA $(1 \mathrm{mM})$ and D-serine $(10 \mu \mathrm{M})$ to the cell soma when the cell was held at $+50 \mathrm{mV}$. Responses were generated by repetitive stimulation $(0.1$ $\mathrm{Hz}$ ), and once a baseline level was established NSC23766 was added to the perfusion.

\section{Results}

\section{Racl is not involved in basal, nor NMDAR-stimulated, CREB} signaling in neurons

To determine whether Rac1 plays a role in CREB signaling, we first examined the effect of Rac1 inhibitor NSC23766 on basal pCREB levels. We found that application of NSC23766 $(100 \mu \mathrm{M})$ to primary cortical neurons for $2 \mathrm{~min}, 5 \mathrm{~min}, 10 \mathrm{~min}, 30 \mathrm{~min}$, or 60 min markedly decreased pCREB levels (Fig. $1 A$, left), and this effect occurred in a dose-dependent manner with saturating effects $\sim 50 \mu \mathrm{M}$ (Fig. 1A, right). We next sought to determine whether Rac1 plays a role in CREB signaling by performing overexpression and RNA interference-based knockdown strategies. To our surprise, we found that overexpressing wild-type Racl protein had no effect on pCREB in neurons (Fig. 1B). Moreover, the expression of a constitutively active Rac1 mutant (Q61L mutation) or a dominant-negative Racl mutant (T17N mutation) protein had no effect on pCREB levels either (Fig. 1B). Finally, knockdown of endogenous Racl using recombinant lentivirus expressing RNAi against Racl (Fig. $1 B$, right) had no effect on the basal pCREB (Fig. 1B). These results provide evidence that NSC23766 decreases basal pCREB in a Racl-independent manner.

Strong NMDAR stimulation ( $>5 \mathrm{~min}$ ) leads to pCREB shutoff (Sala et al., 2000). We found that high-dose NMDA application $(100 \mu \mathrm{M}, 10 \mathrm{~min})$ also led to pCREB shutoff in neurons expressing dominant-negative Rac1 mutant, Rac1 (T17N) (Fig. $1 C)$. However, pretreatment of neurons with NSC23766 (100 $\mu \mathrm{M})$ eliminated the NMDA effect of shutting off pCREB in these Racl (T17N)-expressing neurons (Fig. 1C). Our findings indicate that Racl protein does not play a crucial role for NMDARmediated CREB signaling in neurons.

\section{NSC23766 antagonizes the pCREB signaling activated by both synaptic and extrasynaptic NMDARs}

To help understand how NSC23766 affects pCREB signaling, we determined the effect of NSC23766 compound on pCREB levels in response to synaptic and extrasynaptic NMDAR signaling. Bicuculline application to neurons primarily activates synaptic NMDARs and increases pCREB levels (Hardingham et al., 2002). We replicated this finding by incubating neurons with bicuculline ( $50 \mu \mathrm{M})$ for $40 \mathrm{~min}$ (Fig. 2A, left). Coapplication of NSC23766 $(100 \mu \mathrm{M})$ with bicuculline blocked the increase of pCREB signal evoked by bicuculline application alone (Fig. $2 \mathrm{~A}$, left), suggesting that NSC23766 blocked the synaptic NMDAR-mediated CREB on pathway. High-dose NMDA/glutamate application after bicuculline application has been shown to activate a presumed extrasynaptic NMDAR-mediated signaling mechanism to shut off pCREB (Hardingham et al., 2002). Consistent with this literature, we found that application of high-dose NMDA (100 $\mu \mathrm{M}, 10 \mathrm{~min})$ after $30 \mathrm{~min}$ of bicuculline application $(50 \mu \mathrm{M})$ led to pCREB 
shutoff (Fig. 2A, right). Coapplication of NSC23766 (100 $\mu \mathrm{M})$ with high-dose NMDA $(100 \mu \mathrm{M})$ significantly attenuated the pCREB shutoff (Fig. $2 A$, right), indicating that NSC23766 also blocked extrasynaptic NMDAR-mediated signaling.

NMDAR-mediated signaling also affects ERK1/2 phosphorylation oppositely depending on the locus of NMDAR activation (Ivanov et al., 2006), similar to its action on pCREB. Consistent with this, we found that synaptic NMDAR activation, induced by treatment of neurons with bicuculline $(50 \mu \mathrm{M})$, led to robust increase of phosphor-ERK1/2 (pERK1/2; Fig. 2B, left) while high-dose NMDA $(100 \mu \mathrm{M})$ application afterward blocked it (Fig. $2 B$, right). Interestingly, we found that NSC23766 $(100 \mu \mathrm{M})$ coapplication with bicuculline led to a pERK1/2 level even below the baseline (Fig. 2B, left). Moreover, coapplication of NSC23766 (100 $\mu \mathrm{M}$ ) with high-dose NMDA 30 min after bicuculline application significantly attenuated the NMDA effect on pERK1/2 level (Fig. 2B, right). Our data thus indicate that NSC23766 can inhibit the NMDA effects on both pCREB and pERK1/2 signaling pathways.

\section{NSC23766 converts pCREB shutoff to positive PCREB: potential relevance to glutamate toxicity in stroke}

Reports that NSC23766 treatment prevents cell death in stroke studies (Rex et al., 2009; Raz et al., 2010) prompted us to perform more NSC23766 studies on pCREB signaling in response to bath application of NMDA for the following two reasons: (1) high-dose NMDA bath application likely mimics the massive glutamate accumulation in extracellular space that occurs during stroke and (2) pCREB shutoff in response to strong NMDAR activation is thought to be a leading mechanism of glutamate excitotoxicity in stroke (Hardingham and Bading, 2010). In agreement with a previous report (Sala et al., 2000), we found that mild NMDAR stimulation (20 $\mu \mathrm{M}$ NMDA) led to a pCREB plateau above the basal level (i.e., persistent CREB activation) after initial peak, whereas a high-dose of NMDA $(100 \mu \mathrm{M})$, termed strong NMDAR stimulation, led to a pCREB plateau well below basal level (i.e., persistent pCREB shutoff) after initial pCREB peak (Fig. 3A). These results indicate that bath application of NMDA in cultured neurons activates both synaptic and extrasynaptic NMDAR signaling and the competition between them decides the final equilibrium or plateau pCREB level, i.e., synaptic NMDAR signaling, which activates CREB, dominates when NMDAR is mildly stimulated while extrasynaptic NMDAR signaling, which shuts off CREB, dominates when NMDAR is strongly stimulated.

A

B ${ }^{* *} p<0.01 ;{ }^{* *} p<0.001$.
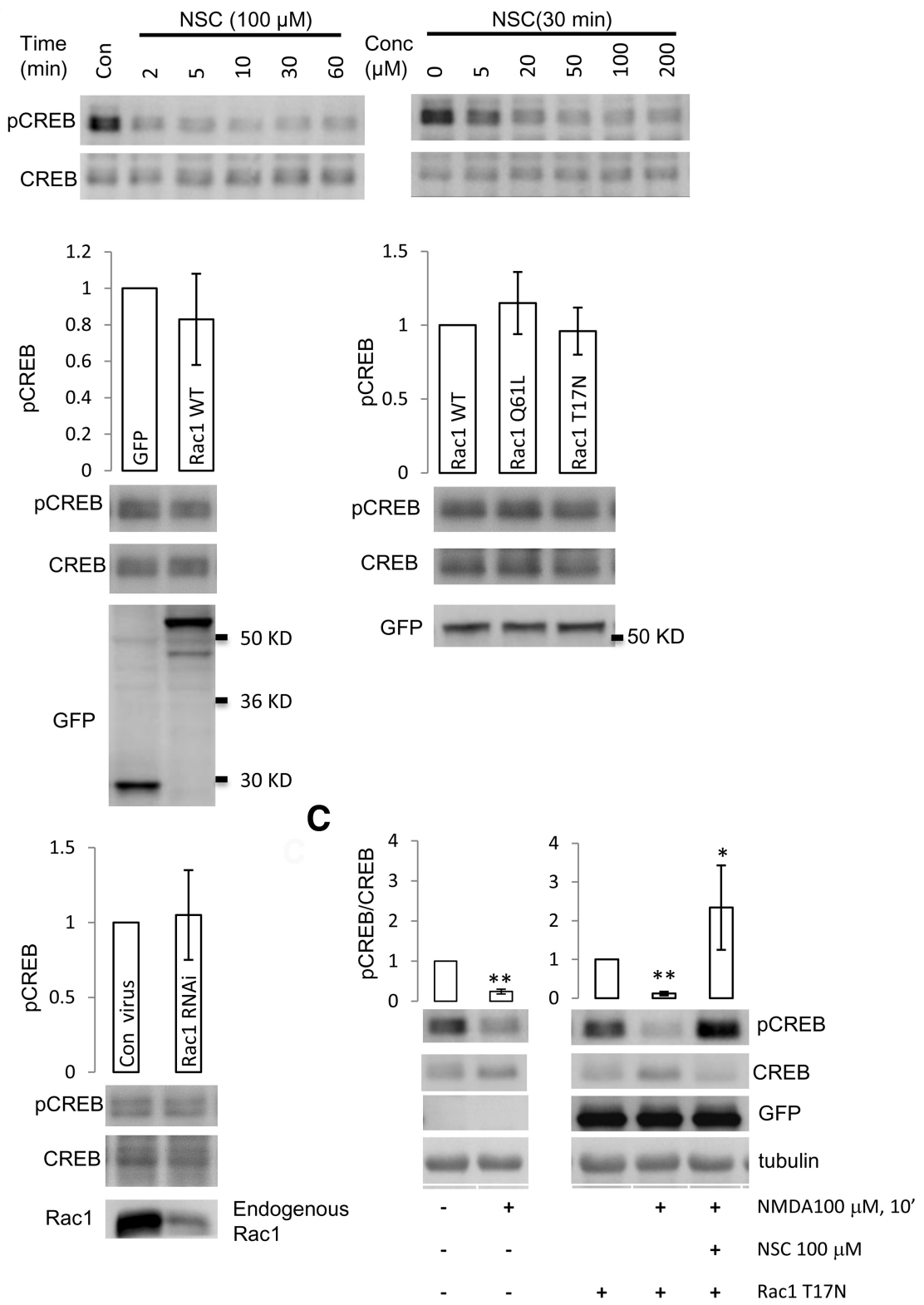

Figure 1. Rac1 is not involved in CREB signaling in neurons. A, Rac1 inhibitor NSC23766 decreases CREB phosphorylation at serine 133 (pCREB) in cortical neurons. Left, Temporal profiles of NSC23766 (100 $\mu \mathrm{M}$ ) on basal pCREB levels. Right, Dose-response effects of NSC23766 (30 min) application on pCREB. B , Expression of EGFP-tagged recombinant Rac1 wild-type (WT; top left), constitutive active (Q61L), and dominant-negative (T17N) Rac1 mutant (top right) or ShRNA against Rac1 (bottom) did not affect pCREB levels. $C$, The inhibition of endogenous Rac1 by overexpression of dominant-negative Rac1 did not affect pCREB shutoff induced by strong NMDAR stimulation, whereas the pretreatment with NSC23766 completely eliminated the NMDA effect in Rac1 T17N overexpressing neurons. One-way ANOVA test (followed by LSD test when there is significance), with $N=3 .{ }^{*} p<0.05$;

Interestingly, we found that NSC23766 antagonizes the NMDA effect on PCREB signaling in response to both strong and mild NMDAR stimulations (Fig. 3B-D). First, pretreatment of neurons with NSC23766 increased the pCREB signal for all durations of high-dose NMDA bath application $(100 \mu \mathrm{M})$, while it decreased the PCREB signal for all durations of the low-dose NMDA bath application (20 $\mu$; Fig. 3B). Simultaneous coapplication of NSC23766 and high-dose NMDA led to pCREB levels above baseline, similar to the effect of low-dose NMDA application alone (Fig. 3C). On the other hand, simultaneous coapplication of NSC23766 and low-dose NMDA led to PCREB plateau 
A

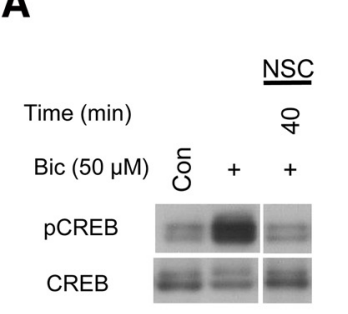

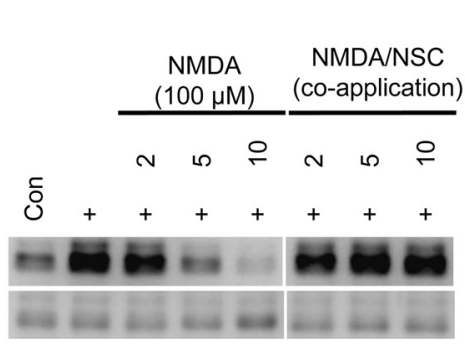

NMDA 5

NMDA $10^{\prime}$
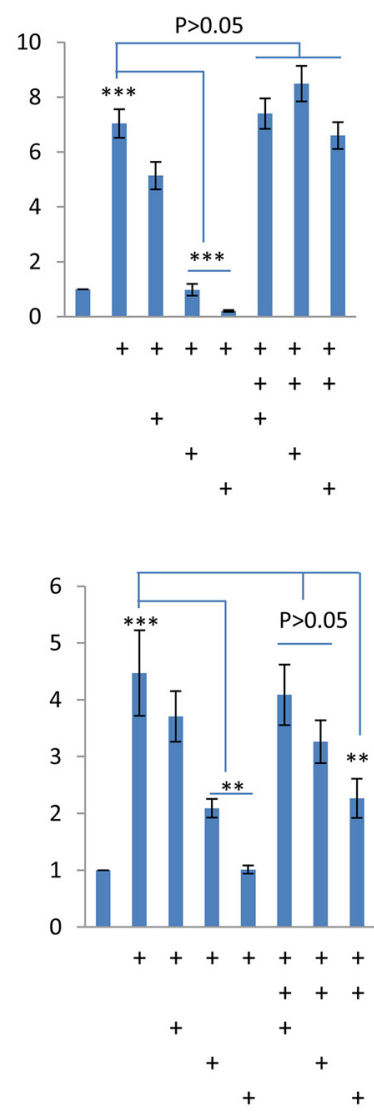

Figure 2. NSC23766 antagonized NMDAR-mediated CREB and Erk1/2 signalings in neurons. $A$, Left, NSC23766 (100 $\mu$ m) coapplication with bicuculline blocked the pCREB increase induced by bicuculline application alone. Right, NSC23766 (100 $\mu \mathrm{m}$ ) coapplication with NMDA (100 $\mu \mathrm{m}$ ) blocked the pCREB shutoff by extrasynaptic NMDAR signaling. Bicuculline treatment (30 min)-induced robust CREB activation was shut off by subsequent application of high-dose NMDA (middle). This pCREB shutoff is prevented when NSC23766 was coapplied together with NMDA. $\boldsymbol{B}$, Similar as in $\boldsymbol{A}$, except that pERK1/2 and ERK1/2 were blotted on duplicate gel/membrane. (Bicuculline was still present during NMDA or NMDA/NSC application in both $\boldsymbol{A}$ and $\boldsymbol{B}$ ). One-way ANOVA (followed by LSD test when there is significance), with $N=3 .^{*} p<0.05$; $^{* *} p<0.01$; ${ }^{* * *} p<0.001$.

below baseline, similar to the effect of high-dose NMDA application alone (Fig. 3C). Finally, $2 \mathrm{~min}$, but not $30 \mathrm{~min}$, post application of NSC23766 relative to NMDA also antagonizes the effects of bath applications of NMDA alone (Fig. $3 D, E$ ). This suggests that pCREB shutoff signaling takes longer than $2 \mathrm{~min}$, but shorter than $30 \mathrm{~min}$, after NMDA application to start. pCREB shutoff signaling is independent of NMDAR activation once it starts. Consistent with previous reports that PP1 (Bito et al., 1996; Sala et al., 2000) and PP2A (Mauna et al., 2011) are the CREB phosphatases, we found that high-dose okadaic acid (200 nM) pretreatment blunted the pCREB shutoff signaling by NMDA (100 $\mu \mathrm{M}$; Fig. $3 F$ ) and also partially occluded NSC23766's inhibitory effect on NMDA-dependent pCREB shutoff (Fig. $3 F$ ). In summary, our results confirmed that (1) NSC23766 antagonizes both synaptic and extrasynaptic NMDAR function in PCREB signaling and (2) NSC23766 can convert the pCREB shutoff during strong NMDAR activation, a mimic of glutamate toxicity in stroke, to positive PCREB, and thus potentially beneficial signaling for neuronal health.

\section{NSC23766 inhibits NMDAR-mediated currents and plasticity} in CA1 pyramidal neurons and cortical neurons

To determine whether NSC23766 can directly act on NMDARs, we examined the effect of NSC23766 perfusion on NMDARmediated currents. We first measured synaptically evoked NMDAR currents in CA1 pyramidal neurons (see Materials and
Methods). Bath application of NSC23766 (100 $\mu \mathrm{M})$ significantly reduced NMDAR-mediated EPSCs (Fig. 4A), in a voltageindependent manner (Fig. $4 A, B$ ), raising the possibility that NSC23766 directly antagonized NMDARs. We also measured NMDA-evoked responses in primary cultured cortical neurons from mouse in response to local NMDA application (see Materials and Methods). NSC23766 perfusion caused significant and reversible suppression of NMDA-evoked currents (Fig. 4C). In contrast, NSC23766 did not affect AMPA receptor-mediated synaptic transmission (Fig. 4D). Finally, we examined the effect of NSC23766 on NMDAR-dependent synaptic plasticity (see Materials and Methods). While LTP was impaired (Fig. 4E), NSC23766 caused a minor reduction, albeit not significant, on LTD (Fig. 4F).

\section{Discussion}

The experiments presented here investigated whether Rac1, a member of the Rho family of GTPases, could play a role in CREB signaling downstream of NMDAR activation. Although Rac1 can couple NMDAR function to cell development (Tolias et al., 2005; Xiao et al., 2013) and that NMDAR is a critical player in CREB signaling (Lyons and West, 2011), our findings reveal that molecular manipulation of Rac1 does not affect CREB signaling either during basal state or after NMDAR activation. Specifically, we showed that neither knockdown of Racl with RNA interference nor expression of either constitutive active or dominant-negative Racl affected basal pCREB levels. Moreover, dominant-negative 
A no NSC treatment
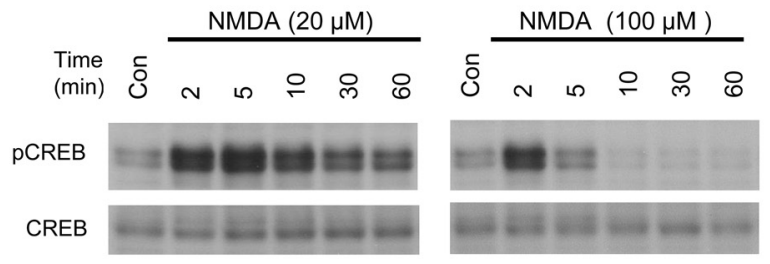

B NSC pretreatment

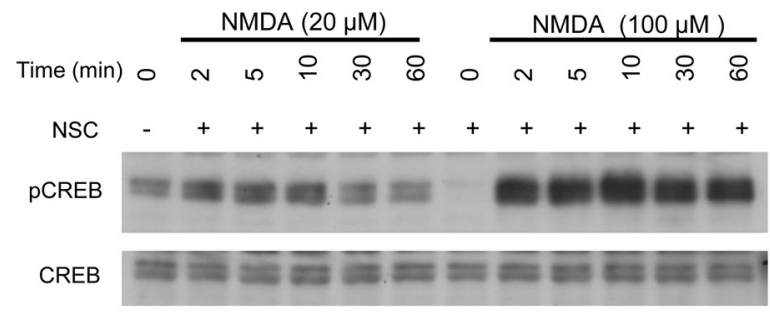

C nSC/NMDA co-application

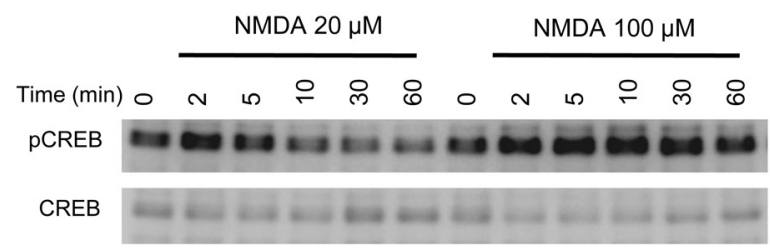

D NSC posttreatment

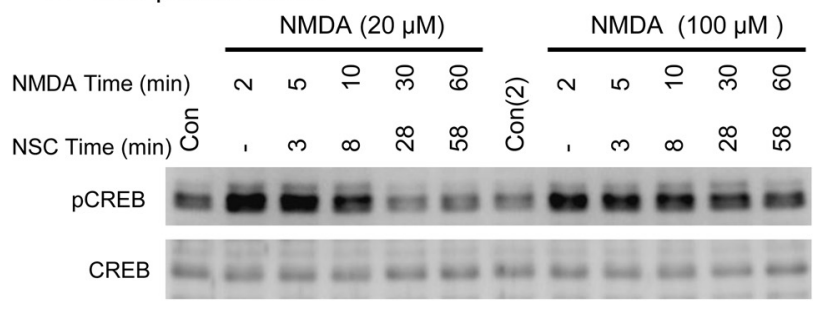

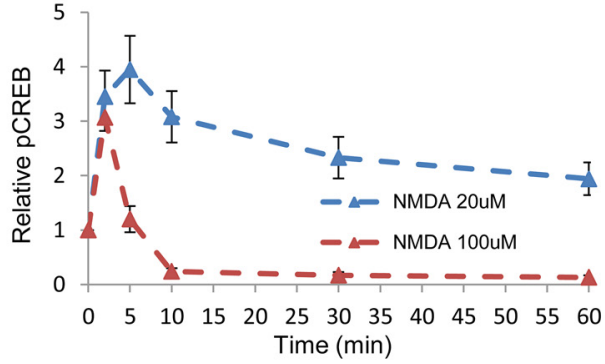
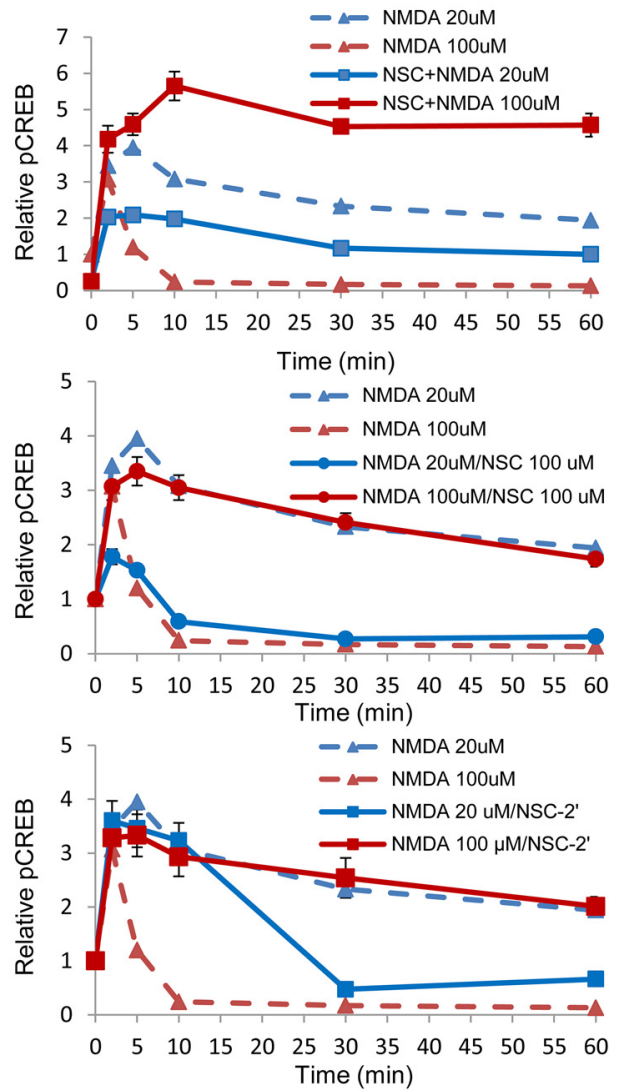

E

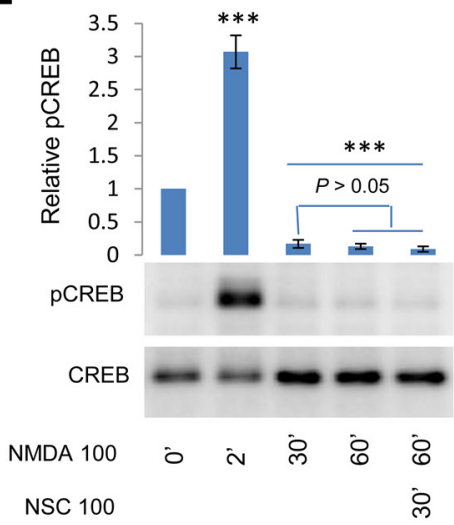

$\mathbf{F}$

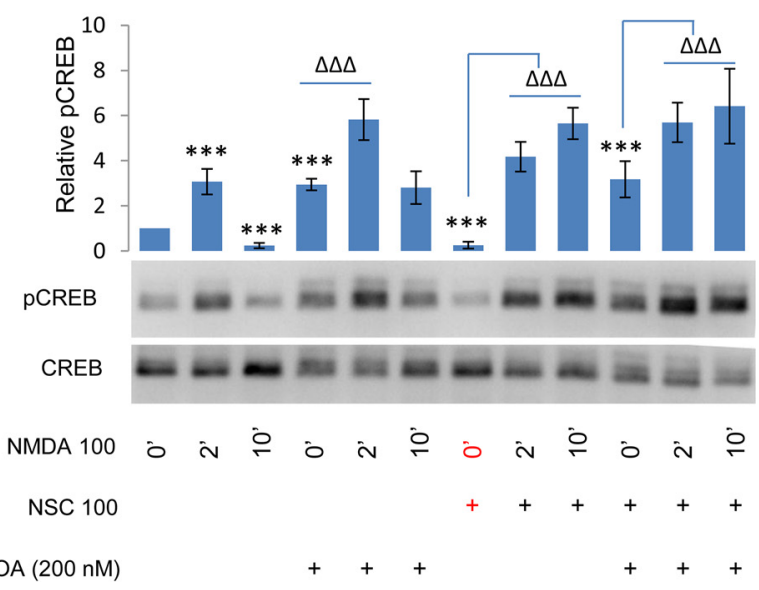

Figure 3. NSC23766 antagonized NMDAR-mediated CREB signaling in neurons. A, Time course of mild and strong NMDA stimulation on pCREB levels in 2-week-old neurons. Pretreatment for 30 $\min (\boldsymbol{B})$, simultaneous $(\boldsymbol{C})$, or post treatment ( 2 min after NMDA, $\boldsymbol{D} ; 30$ min after NMDA, $\boldsymbol{E}$ ) of neurons with NSC23766 relative to NMDA bath application antagonizes the $p$ CREB signal induced by bath application of NMDA alone ( $p<0.05$, compared with NMDA treatment alone). $\boldsymbol{F}$, Pretreatment of high-dose okadaic acid (OA; $200 \mathrm{nM})$, a PP1/PP2A inhibitor, occludes most of the NSC23766 effect on high-dose NMDA-dependent CREB phosphorylation. One-way ANOVA (followed by LSD test when there is significance), with $N=3 .{ }^{* * *}$ and $\Delta \Delta \Delta: p<0.001$. 
A
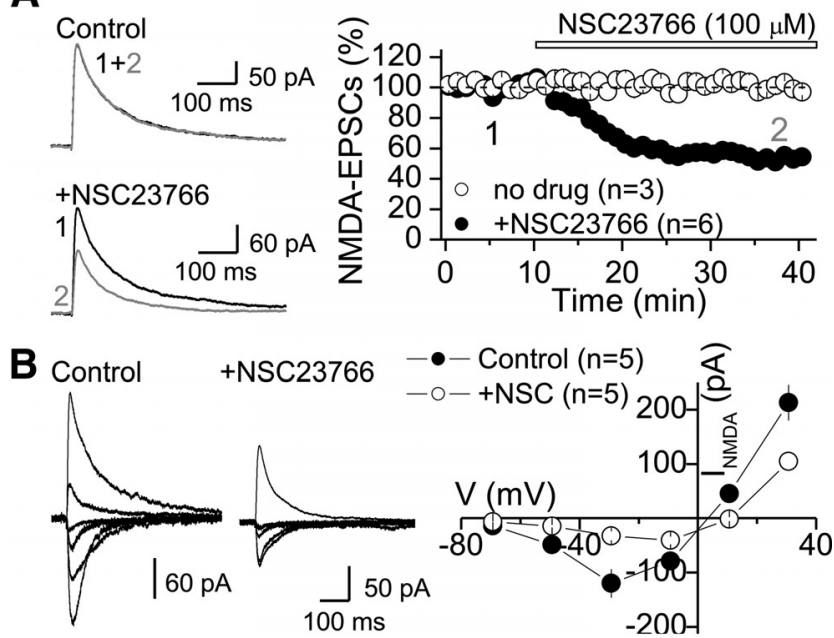

C
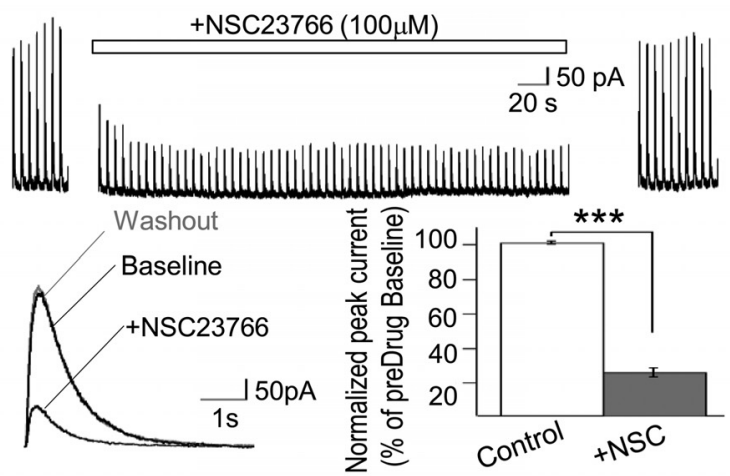

D
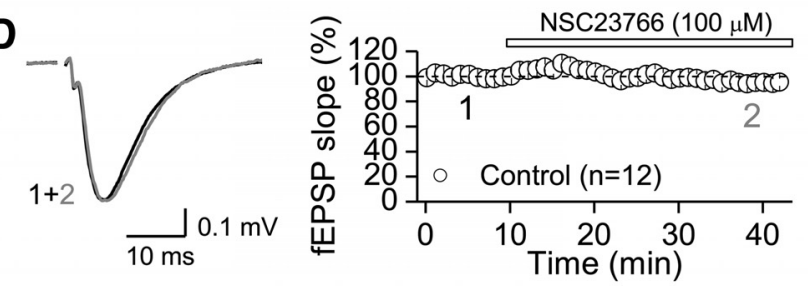

E
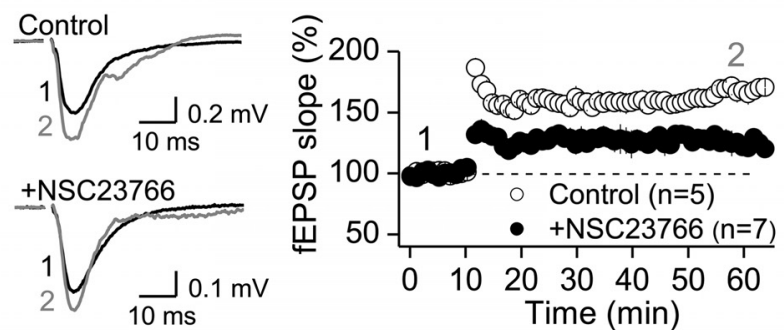

$\mathbf{F}$
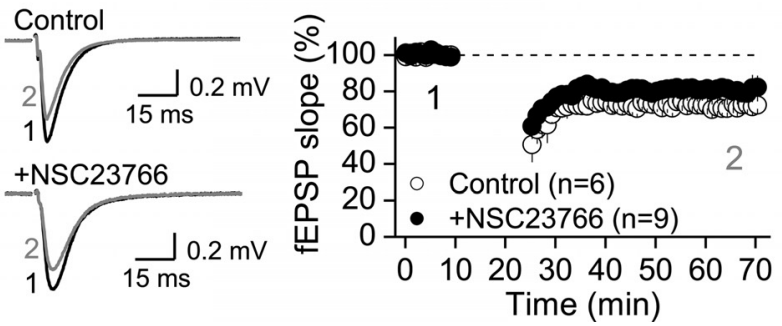

Figure 4. NSC23766 inhibits NMDAR-mediated currents and plasticity in hippocampal and cortical neurons. $A$, Perfusion of NSC23766 decreases NMDAR-mediated EPSC in CA1 pyramidal neurons in acute rat brain slices ( $54.8 \pm 2.0 \%$ of baseline; $p<0.001$, paired $t$ test; $n=6$ ). Synaptic responses in naive slices are superimposed for comparison purposes $(n=3)$. $\boldsymbol{B}$, NSC23766 decreases NMDAR-mediated EPSCs at all voltages tested. NMDA I/V plots were performed before and after NSC23766 in the same neuron $(n=5)$. C, NSC23766 also inhibits
Racl expression did not affect bath NMDA effect on pCREB levels, thereby suggesting that Racl does not play a role in NMDAR stimulation-induced CREB signaling.

We show here for the first time that NSC23766 inhibits basal CREB activation while also blocking the effects of NMDA bath application on CREB signaling. The most conclusive data on the Racl independence of NSC23766 effect on CREB signaling is that NSC23766 can, but expression of dominant-negative Rac1 mutant cannot, block the effect of high-dose NMDA bath application on pCREB shutoff (Fig. 1C). Glutamate transporter malfunction during stroke leads to massive glutamate accumulation in extracellular space including extrasynaptic sites and causes glutamate excitotoxicity. High-dose NMDA bath application leading to pCREB shutoff is one leading cellular mechanism of glutamate excitotoxicity in stroke (Hardingham and Bading, 2010). So the function of NSC23766 in blocking the pCREB shutoff induced by high-dose NMDA application would indicate that NSC23766 could prevent cell death in the glutamate toxicity model of stroke.

Our second novel finding is that NSC23766, a putative Rac1 selective inhibitor, has an interesting off-target effect on NMDARs. Our electrophysiological studies provide strong evidence that NSC23766 perfusion directly attenuates NMDARmediated currents in both slice preparation and primary neurons, strongly supporting the idea that the NSC23766 effects on pCREB and pERK1/2 are likely mediated by its direct effect on NMDARs, independent of its Racl function. A previous study reported that NSC23766, presumably by blocking Rac1 activation, impaired NMDAR-dependent LTP and LTD in the CA1 area of the hippocampus (Martinez and Tejada-Simon, 2011). In our hands, NSC23766 impaired LTP, but had minimal effect on LTD. While the exact effect of NSC23766 on NMDAR function remains elusive, the rapid NSC23766 antagonism on NMDARmediated currents suggests a direct action toward the NMDAR complex, rather than a secondary effect on the trafficking of NMDARs downstream of Rac1 inhibition. Further experiments to test the exact mechanism underlying NSC23766 effect on NMDARs are needed.

Since the original report (Gao et al., 2004) that characterized NSC23766 as a selective Rac1 inhibitor, NSC23766 has been widely used as a specific Racl inhibitor in the neuroscience field (Kim et al., 2007; Rex et al., 2009; Zhang et al., 2009; Raz et al., 2010; Martinez and Tejada-Simon, 2011). For example, NSC23766 administration significantly attenuates oxidative stress damage to CA1 regions after ischemia (Raz et al., 2010) and apoptotic neuronal cell death following global cerebral ischemia (Zhang et al., 2009). While this neuroprotective function of NSC23766 compound is attributed to its inhibitory property on Rac1 (Zhang et al., 2009; Raz et al., 2010), our results open the possibility that NSC23766 may achieve neuroprotection by directly antagonizing NMDAR function and blocking pCREB shutoff in glutamate toxicity.

In summary, we found that Racl does not play a role in CREB signaling and that Rac1 inhibitor NSC23766 has additional function in reducing NMDAR-mediated currents. Our study strongly suggests that NSC23766 conversion to CREB activation via its

$\leftarrow$

NMDA-elicited currents $\left(V_{h}=+50 \mathrm{mV}\right)$ in mouse primary cortical neurons $(26.37 \pm 2.60 \%$ of baseline; $p=0.002$, paired $t$ test, $n=3$ ). Top, Representative experiment. Bottom left, NMDAmediated responses. Bottom right, Summary plot. D, NSC23766 does not affect AMPA receptormediated transmission ( $95.7 \pm 2.9 \%$ of baseline; $p>0.05$, paired $t$ test; $n=12$ slices). $\boldsymbol{E}, \boldsymbol{F}$, Pretreatment with NSC23766 for 30 min attenuates LTP (control: $n=5 ;+N S C 23766: n=7$; $p=0.0032$, ANOVA), but not LTD (control: $n=6 ;+\operatorname{NSC23766:~} n=9 ; p=0.079$ ANOVA). 
NMDAR antagonistic function, from pCREB shutoff in glutamate toxicity, underlies the beneficial effect of NSC23766 in cell health in stroke model studies. The biological effects of NSC23766 in neurons may first arise from its surface NMDAR antagonistic function instead of cytosolic Racl inhibition, because NSC23766 treatment will target surface NMDARs before crossing plasma membrane to inhibit Racl. Studies using NSC23766 in neurons require further validation regarding the presumed Racl functions.

\section{References}

Bito H, Deisseroth K, Tsien RW (1996) CREB phosphorylation and dephosphorylation: a $\mathrm{Ca}(2+)$ - and stimulus duration-dependent switch for hippocampal gene expression. Cell 87:1203-1214. CrossRef Medline

Chávez AE, Chiu CQ, Castillo PE (2010) TRPV1 activation by endogenous anandamide triggers postsynaptic long-term depression in dentate gyrus. Nat Neurosci 13:1511-1518. CrossRef Medline

Colombo PJ, Brightwell JJ, Countryman RA (2003) Cognitive strategyspecific increases in phosphorylated cAMP response element-binding protein and c-Fos in the hippocampus and dorsal striatum. J Neurosci 23:3547-3554. Medline

Deisseroth K, Tsien RW (2002) Dynamic multiphosphorylation passwords for activity-dependent gene expression. Neuron 34:179-182. CrossRef Medline

Gao Y, Dickerson JB, Guo F, Zheng J, Zheng Y (2004) Rational design and characterization of a Rac GTPase-specific small molecule inhibitor. Proc Natl Acad Sci U S A 101:7618-7623. CrossRef Medline

Haditsch U, Leone DP, Farinelli M, Chrostek-Grashoff A, Brakebusch C, Mansuy IM, McConnell SK, Palmer TD (2009) A central role for the small GTPase Racl in hippocampal plasticity and spatial learning and memory. Mol Cell Neurosci 41:409-419. CrossRef Medline

Hall BJ, Ripley B, Ghosh A (2007) NR2B signaling regulates the development of synaptic AMPA receptor current. J Neurosci 27:13446-13456. CrossRef Medline

Hardingham GE, Bading H (2010) Synaptic versus extrasynaptic NMDA receptor signalling: implications for neurodegenerative disorders. Nat Rev Neurosci 11:682-696. CrossRef Medline

Hardingham GE, Fukunaga Y, Bading H (2002) Extrasynaptic NMDARs oppose synaptic NMDARs by triggering CREB shut-off and cell death pathways. Nat Neurosci 5:405-414. Medline

Hou H, Sun L, Siddoway BA, Petralia RS, Yang H, Gu H, Nairn AC, Xia H (2013) Synaptic NMDA receptor stimulation activates PP1 by inhibiting its phosphorylation by Cdk5. J Cell Biol 203:521-535. CrossRef Medline

Ivanov A, Pellegrino C, Rama S, Dumalska I, Salyha Y, Ben-Ari Y, Medina I (2006) Opposing role of synaptic and extrasynaptic NMDA receptors in regulation of the extracellular signal-regulated kinases (ERK) activity in cultured rat hippocampal neurons. J Physiol 572:789-798. CrossRef Medline
Kandel ER (2012) The molecular biology of memory: cAMP, PKA, CRE, CREB-1, CREB-2, and CPEB. Mol Brain 5:14. CrossRef Medline

Karpova A, Mikhaylova M, Bera S, Bär J, Reddy PP, Behnisch T, Rankovic V, Spilker C, Bethge P, Sahin J, Kaushik R, Zuschratter W, Kähne T, Naumann M, Gundelfinger ED, Kreutz MR (2013) Encoding and transducing the synaptic or extrasynaptic origin of NMDA receptor signals to the nucleus. Cell 152:1119-1133. CrossRef Medline

Kim MJ, Futai K, Jo J, Hayashi Y, Cho K, Sheng M (2007) Synaptic accumulation of PSD-95 and synaptic function regulated by phosphorylation of serine-295 of PSD-95. Neuron 56:488 -502. CrossRef Medline

Lyons MR, West AE (2011) Mechanisms of specificity in neuronal activityregulated gene transcription. Prog Neurobiol 94:259-295. CrossRef Medline

Martinez LA, Tejada-Simon MV (2011) Pharmacological inactivation of the small GTPase Racl impairs long-term plasticity in the mouse hippocampus. Neuropharmacology 61:305-312. CrossRef Medline

Mauna JC, Miyamae T, Pulli B, Thiels E (2011) Protein phosphatases 1 and $2 \mathrm{~A}$ are both required for long-term depression and associated dephosphorylation of cAMP response element binding protein in hippocampal area CA1 in vivo. Hippocampus 21:1093-1104. CrossRef Medline

Raz L, Zhang QG, Zhou CF, Han D, Gulati P, Yang LC, Yang F, Wang RM, Brann DW (2010) Role of Racl GTPase in NADPH oxidase activation and cognitive impairment following cerebral ischemia in the rat. PLoS One 5:e12606. CrossRef Medline

Rex CS, Chen LY, Sharma A, Liu J, Babayan AH, Gall CM, Lynch G (2009) Different Rho GTPase-dependent signaling pathways initiate sequential steps in the consolidation of long-term potentiation. J Cell Biol 186:85-97. CrossRef Medline

Sala C, Rudolph-Correia S, Sheng M (2000) Developmentally regulated NMDA receptor-dependent dephosphorylation of cAMP response element-binding protein (CREB) in hippocampal neurons. J Neurosci 20:3529-3536. Medline

Siddoway BA, Altimimi HF, Hou H, Petralia RS, Xu B, Stellwagen D, Xia H (2013) An essential role for inhibitor-2 regulation of protein phosphatase-1 in synaptic scaling. J Neurosci 33:11206-11211. CrossRef Medline

Tolias KF, Bikoff JB, Burette A, Paradis S, Harrar D, Tavazoie S, Weinberg RJ, Greenberg ME (2005) The Rac1-GEF Tiam1 couples the NMDA receptor to the activity-dependent development of dendritic arbors and spines. Neuron 45:525-538. CrossRef Medline

Wang CC, Held RG, Chang SC, Yang L, Delpire E, Ghosh A, Hall BJ (2011) A critical role for GluN2B-containing NMDA receptors in cortical development and function. Neuron 72:789-805. CrossRef Medline

Xiao L, Hu C, Yang W, Guo D, Li C, Shen W, Liu X, Aijun H, Dan W, He C (2013) NMDA receptor couples Rac1-GEF Tiam1 to direct oligodendrocyte precursor cell migration. Glia 61:2078-2099. CrossRef Medline

Zhang QG, Wang R, Han D, Dong Y, Brann DW (2009) Role of Racl GTPase in JNK signaling and delayed neuronal cell death following global cerebral ischemia. Brain Res 1265:138-147. CrossRef Medline 